\title{
Efectos de un compost enriquecido con microorganismos eficientes sobre la germinación de semillas recalcitrantes de Artocarpus altilis (Parkinson) Fosberg y Theobroma cacao L. \\ Effects of enriched compost with efficient microorganisms on the germination of recalcitrant seeds of breadfruit (Parkinson) Fosberg and Theobroma cacao $\mathrm{L}$ \\ Yánez Yánez Wilfrido ${ }^{1 *}$, Villacís-Aldaz Luis Alfredo ${ }^{1}$, León-Gordón Olguer Alfredo', Velástegui-Espín Giovanny Patricio ${ }^{1}$, López-Villacís Isabel Cristina ${ }^{1}$, Cruz-Tobar Saúl Eduardo $^{1}$
}

\begin{tabular}{l} 
Datos del Articulo \\
\hline 19acultad de Ciencias Agropecuarias \\
Universidad Técnica de Ambato, Tungu- \\
rahua, Ecuador. \\
Casilla postal: $18-01-334$. \\
Telf: (+593)032872630-0985471191 \\
aw.yanez@uta.edu.ec \\
\hline ao.leon@uta.edu.ec \\
gp.velastegui@uta.edu.ec \\
ic.lópez@uta.edu.ec \\
se.cruz@uta.edu.ec \\
*Dirección de contacto: \\
Wilfrido Yánez-Yánez \\
Facultad de Ciencias Agropecuarias. \\
Universidad Técnica de Ambato. \\
Tungurahua, Ecuador. \\
Casilla postal: $18-01-334$. \\
Telf: $(+593) 032872630-0987010062$ \\
E-mail: aw.yanez@uta.edu.ec \\
\hline
\end{tabular}

Palabras clave:
Compost,
levaduras,
ácido láctico,
germinación,
emergidas.

J Selva Andina Biosph. 2016; 4(2):100-108.

\section{Historial del artículo.}

Recibido mayo, 2016.

Devuelto septiembre 2016

Aceptado septiembre, 2016

Disponible en línea, noviembre 2016

Editado por:

Selva Andina

Research Society

\section{Key words:}

Compost,

yeast,

lactic acid,

lactic acid,

emerged.

\section{Resumen}

La investigación se realizó en el Centro de Investigación, Postgrado y Conservación Amazónica (CIPCA) de la Universidad Estatal Amazónica, Ecuador. El propósito fue evaluar el efecto de un compost enriquecido en la germinación, días de emergencia y días al trasplante de dos especies de origen tropical Artocarpus altilis y Theobroma cacao. El campo experimental está ubicado en la provincia de Napo, a $1^{\circ} 16^{\prime}$ y $36^{\prime \prime} \mathrm{S}$ y $77^{\circ} 58^{\prime}$ y $76^{\prime \prime} \mathrm{W}$, a una altitud de $556 \mathrm{msnm}, 556$ msnm con temperatura entre 18 y $32{ }^{\circ} \mathrm{C}$, precipitación promedio anual por encima de $3000 \mathrm{~mm}$ y humedad relativa entre 87 y $89 \%$. Se aplicó un diseño experimental con cinco tratamientos en bloques completamente al azar con tres repeticiones. Los tratamientos consistieron de compost (1) con EM, (2) con bacterias del ácido láctico (LAB), (3) con levaduras comerciales, con levaduras adquiridas de forma comercial en el supermercado (4) Mezcla de los anteriores y (5) solo compost, sin ningún adicionamiento de microrganismos, testigo. Se determinó que la mezcla de compost (T4) en su conjunto, tiene el mayor efecto sobre los parámetros de las semillas. En la germinación se comprobó que en dos de los tratamientos T3 y T4 disminuyeron significativamente el número de días a la germinación, en relación al testigo, 33 días en Artocarpus altilis y 13 días en Teobroma cacao. El número de plantas emergidas, aumento en ambas especies con la adición de EM, LAB y levadura comercial (T4). Se reporta también una reducción en los días al trasplante de las especies estudiadas siendo significativa particularmente en cacao. Igualmente se determinó que la mayor carga microbiológica correspondió a las bacterias.

(C) 2016. Journal of the Selva Andina Biosph. Bolivia. Todos los derechos reservados.

Abstract

The research was conducted at the Center for Research, Graduate Studies and Amazon Conservation (CIPA) of the Amazon State University, Ecuador. The purpose was to assess the effect of enriched compost Germination, day and day's emergency transplantation two species of tropical origin and breadfruit Theobroma cacao. The experimental field is located in the province of Napo, 16'-1 and $\mathrm{S}$ and $77^{\circ} 36^{\prime \prime} 58^{\prime} \mathrm{y} 76^{\prime \prime} \mathrm{W}$, at an altitude of 556 meters, with temperature between 18 and $320 \mathrm{C}$, average annual precipitation above $3000 \mathrm{~mm}$ and relative humidity between 87 and $89 \%$. An experimental design with five treatments in a randomized complete block design with three replications was applied. Treatments consisted of compost (1) MS, (2) lactic acid bacteria, (3) commercial yeast, (4) a combination thereof and (5) only compost, witness. It was determined that the compost mixture (T4) as a whole, has the greatest effect on the parameters of the seeds. Germination was found that in two of the T3 and T4 treatments significantly decreased the number of days to germination, in relation to the witness, 33 days and 13 days Artocarpus altilis, Theobroma cacao. The number of emerged plants, increase in both species with the addition of EM, lactic acid bacteria and commercial yeast (T4). a reduction is also reported in the days to transplant species studied to be significant particularly in cocoa. It was also determined that most bioburden corresponded to bacteria. 


\section{Introducción}

Las especies del genero Artocarpus (Moraceae) son conocidas por ocupar una amplia variedad de nichos ecológicos siendo diversas y numerosas, especialmente en ecosistemas forestales (Jagpat \& Batak 2010). Las plantas de Artocarpus ofrecen múltiples ventajas como cultivos multipropósitos en la producción de frutos y madera (Jagpat \& Batak 2010). $\mathrm{Su}$ crecimiento está restringido a los ecosistemas de bosques siempre húmedos tropicales por debajo de los 1000 msnm. Artocarpus altilis (Parkinson) Fosberg (frutepan), es un árbol de mediano tamaño, ampliamente cultivado en los trópicos como material de construcción, suplemento de alimento animal, sus hojas son usadas frecuentemente como tratamiento de la cirrosis hepática, hipertensión y diabetes (Wang et al. 2006). Sus semillas son de color marrón, redondeadas, de 2-3 cm (0.8-1.2 in) de longitud por 1-1.5 cm $(0.4-0.6 \mathrm{~cm})$ de diámetro, sin endospermo, con germinación hipogea. Se han descrito hasta 500 semillas por fruto, recubiertas de una fina membrana blanquecina. Estas son recalcitrantes y pueden ser almacenadas hasta un mes en condiciones de humedad y temperatura fría (Ragone 2006). A. altilis ha sido estudiado por su valor como un cultivo para la seguridad alimentaria (RobertsNkrumah \& Legall 2013). Su importancia radica en su contenido nutricional y su alta productividad.

Algunos estudios recientes (Broomes et al. 2009 y Jones et al. 2011) han reportado que el contenido de carbohidratos en la harina oscila entre $61 \%$ a $84 \%$, siendo excelente fuente de vitaminas A, B con minerales que incluyen potasio, calcio, aminoácidos, ácidos grasos esenciales y fibras. La producción de frutepan en algunos países está asociada con sistemas agroforestales, principalmente en la producción 101 de cacao (Theobroma cacao L.), debido a que aporta sombra, aumentando la longevidad y productividad de las plantas de cacao (Fennah 1957). Changrun \& Wendell (2009), indicaron que las semillas de $T$. cacao muestran igualmente un carácter recalcitrante, siendo muy susceptibles a la desecación, lo que convierte en un obstáculo para su conservación por la pérdida rápida de viabilidad.

La agricultura tradicional ha buscado acrecentar la producción agrícola mediante el manejo del agua, nutrientes, control de malezas, insectos y organismos fitopatógenos. En años recientes, se han buscado prácticas agrícolas que apuntan a utilizar los insumos agrícolas en forma dirigida y controlada en el manejo integrado de plagas y enfermedades (Pedraza et al. 2010). Se ha evaluado la diversidad genética y bioquímica de los microorganismos rizosféricos, su caracterización en relación a la fijación biológica del nitrógeno atmosférico, solubilización de fosfatos, aporte de nutrientes, producción de hormonas vegetales, de sustancias capaces de captar hierro (De Felipe 2004). También se ha incluido la selección de los microorganismos eficientes en experimentos de inoculación en condiciones ambientales controladas de laboratorio, invernadero, campo, al igual que el estudio de costos de producción con la aplicación de microorganismos vs fertilizantes químicos en cultivos de interés agrícola (Elein et al. 2005. García de Salamone (2010) indican que diferentes manejos agrícolas modifican la biodiversidad y alteran la estructura de las comunidades microbiológicas del suelo. Todo esto, ha llevado a la implementación de prácticas de manejo, que garanticen un balance positivo de nutrientes, el uso eficiente de los recursos del sistema suelo-planta, disminuyendo 
la degradación de la materia orgánica (Grandy 2006). La utilización de microorganismos benéficos ha tenido una amplia difusión en los últimos años, debido a su efecto positivo sobre el rendimiento de muchos cultivos en distintas situaciones, a la factibilidad de permitir desarrollar una agricultura orgánica (Caballero-Mellado et al.1992, Cassán \& García de Salamone 2008). La incorporación de organismos seleccionados por sus funciones en diversos procesos que contribuyan a la implantación, desarrollo, producción de cultivos, siendo una alternativa que permite lograr aumentos en el crecimiento radical. Así se favorece la exploración del suelo, se mejora la accesibilidad al agua, nutrientes limitantes para los cultivos. (Benzing 2001). Como consecuencia, se reducen procesos de pérdida de nutrientes móviles, se atenúan períodos de moderado estrés hídrico, se logra mantener tasas de crecimiento activo del cultivo mejorando su capacidad fotosintética (Díaz-Zorita \& Fernández 2009). Una clasificación más específica de los microorganismos benéficos ha sido sugerida por Higa (2013) quien hace referencia a los "Microorganismos Efectivos" o EM. Un EM consiste en un cultivo mixto de microorganismos benéficos, de ocurrencia natural, que pueden ser aplicados como inoculantes para incrementar la diversidad microbial de los suelos, plantas, así mismo, bacterias ácido lácticas, levaduras y un número más pequeño de bacterias fotosintéticas. Todos estos compatibles mutuamente unos con otros, capaces de coexistir en un cultivo líquido. Algunas investigaciones han arrojado que la inoculación de cultivos con EM pueden mejorar la calidad del suelo, el crecimiento, producción y calidad de los cultivos (Campo-Martínez et al. 2014), Navia-Cuetia et al. 2013).

El objetivo de este estudio fue evaluar el efecto de un compost enriquecido en semillas recalcitrantes de A. altilis (Parkinson) Fosberg y T. cacao L.

\section{Materiales y métodos}

El estudio se llevó a cabo en el Centro de Investigación, Postgrado y Conservación Amazónica CIPCA, ubicado a $1^{\circ} 16^{\prime} 39^{\prime \prime} \mathrm{S}, 77^{\circ} 58^{\prime} 16^{\prime \prime} \mathrm{W}$, a una altitud de $556 \mathrm{msnm}$, con temperatura oscilante entre $18^{\circ} \mathrm{C}$ y $32^{\circ} \mathrm{C}$ y precipitación promedio anual que supera los $3000 \mathrm{~mm}$. La humedad relativa está entre el 87 a $89 \%$.

Tabla 1 Conformación de los tratamientos

\begin{tabular}{|c|c|c|c|c|}
\hline $\mathrm{T} 1$ & $\mathrm{~T} 2$ & T3 & $\mathrm{T} 4$ & T5 \\
\hline $\begin{array}{c}\text { EM capturados } \\
1 \mathrm{Kg} / 50 \mathrm{~L} \text { de solución } \\
\text { de melaza al } 10 \%\end{array}$ & $\begin{array}{c}\text { Bacterias del ácido } \\
\text { láctico (LAB) } 1 \mathrm{Kg} / 50 \\
\text { L de solución de mela- } \\
\text { za al } 10 \%\end{array}$ & $\begin{array}{l}\text { Levadura comercial } \\
1 \mathrm{Kg} / 50 \mathrm{~L} \text { de solución } \\
\text { de melaza al } 10 \%\end{array}$ & $\begin{array}{c}\text { Mezcla T1,T2 y T3 } \\
1 \mathrm{Kg} \text { de mezcla de los } \\
\text { tres tratamientos/ } 50 \mathrm{~L} \\
\text { de solución de melaza } \\
\text { al 10\% }\end{array}$ & Compost sin adiciones \\
\hline
\end{tabular}

Los suelos del sector están formados predominantemente por sedimentos de arcilla y areniscas, ligeramente gredoso, de poco drenaje, poco profundos, con topografía principalmente plana. Según Cañadas-Cruz (1988), esta región corresponde a la formación ecológica bosque húmedo pluvial pre montano (bhp.PM) y bosque muy húmedo pluvial pre montano (bmhp.PM), (GAD, Santa Clara 2009) (Diagnóstico Ecoturístico del Cantón Santa Clara. Se aplicaron cinco tratamientos (tabla1) con tres repeticiones cada uno, en un diseño de bloques completamente al azar.

Para la elaboración del compost utilizó estiércol de ganado vacuno y pasto gramalote picado, en una 
proporción de 70 - 30, para regular la relación C/N se incorporó cascarilla de arroz. En cada unidad experimental (cajón de $0.05 \mathrm{~m}$ de alto por $1 \mathrm{~m}$ de largo y por $1 \mathrm{~m}$ de ancho) se colocaron $0.05 \mathrm{~m}^{3} \mathrm{de}$ compost.

Para la captura de microorganismos - EM, (T1) se siguió la metodología reportada por CampoMartínez et al. (2014). Se inició con la preparación del sustrato, que consistió en la cocción de $1 \mathrm{~kg}$ de arroz sin sal durante $15 \mathrm{~min}$, hasta obtener una consistencia semiblanda, se repartió en 100 vasos desechables de 14 onzas, se cubrieron los vasos con tela de nylon y se aseguraron con cauchos de goma, los que fueron llevados a cada sitio seleccionado, siguiendo los criterios de presencia de hojarasca y no intervención del hombre, estos fueron colocados a $3 \mathrm{~cm}$ de profundidad, permitiendo la colonización de los organismos en el sustrato. Al cabo de ocho días los capturadores fueron recolectados y diluidos en una solución madre de melaza al $10 \%$ mas $50 \mathrm{~g}$ de pasta de soya.

Tabla 2 Disponibilidad de macronutrientes (\%), micronutrientes (ppm), materia orgánica (\%), humedad (\%), conductividad eléctrica $(\mathrm{mmhos} / \mathrm{cm})$ y pH del compost en los diferentes tratamientos

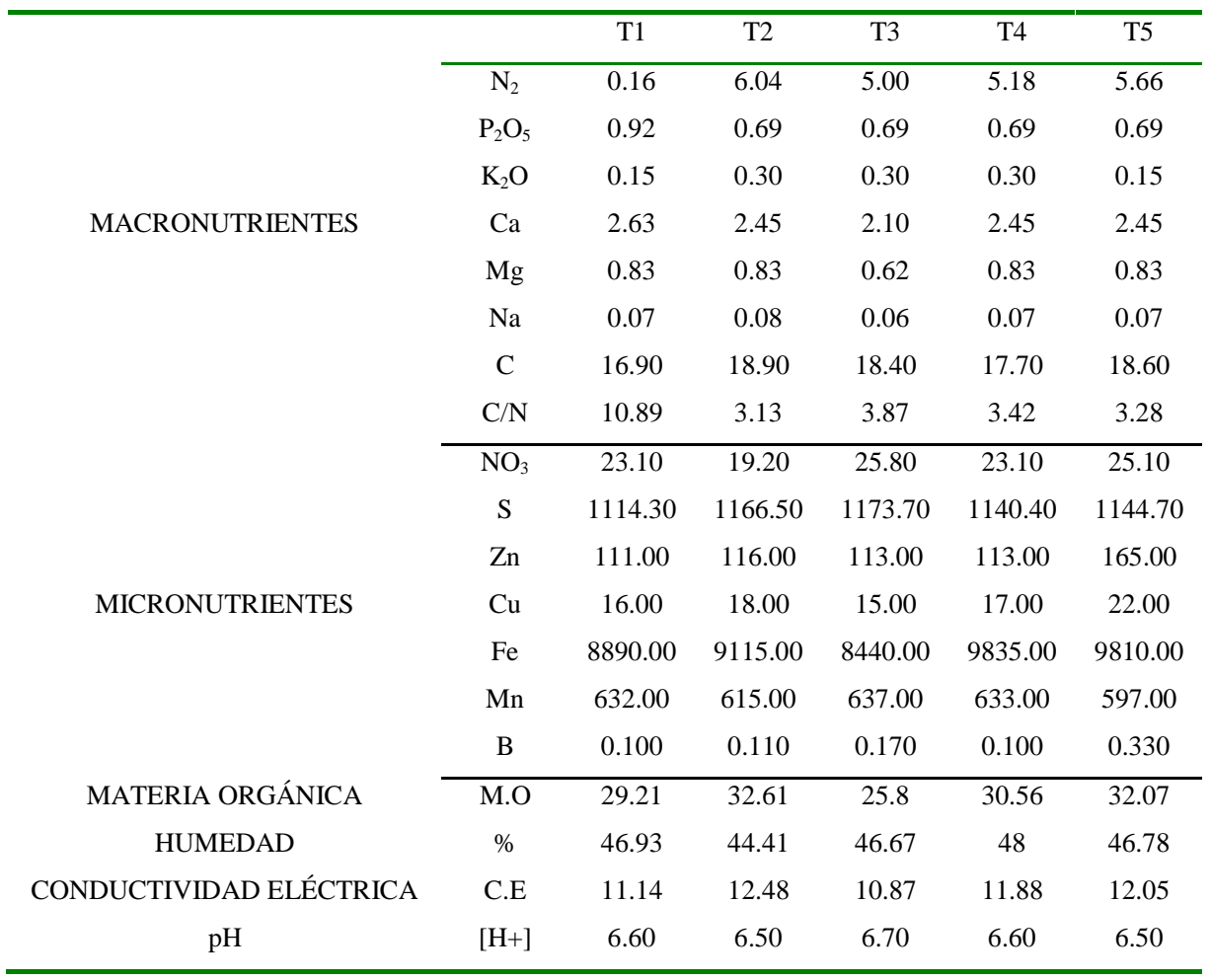

Para obtener bacterias del ácido láctico (LAB) (T2), se colocaron $10 \mathrm{~L}$ de leche en un recipiente con capacidad para 20 L, se dejó en descomposición por espacio de 8 días, bajo condiciones ambientales de la zona, luego de este tiempo las bacterias obtenidas, se colocaron en una solución madre de melaza 103 más leche al $10 \%$ respectivamente y se dejaron por 8 días más en condiciones ambientales.

Se analizaron las características físicas, químicas y microbiológicas del compost inoculado con los EM de cada una de los tratamientos, a los ocho días de comenzar el experimento. Las sub-muestras de 
compost fueron tomadas en diferentes secciones del lote y con la homogenización se conformó una muestra compuesta, para su análisis físico-químico y biológico. Por razones de costos no se pudo analizar las 15 submuestras, sino una muestra compuesta, siguiendo la recomendación de Pérez et al. (2008) (tablas 2 y 3).

Se identificaron los microorganismos presentes y se contabilizó el número de organismos vivos en el compost por el método del hematocymetro y de plato.

\section{Resultados}

La tabla 3 muestra las características físicas y químicas del compost de acuerdo a cada tratamiento. En valores absolutos, se puede observar que todos los valores son similares entre los diferentes tratamientos, un valor promedio de $\mathrm{N}(0.16)$ muy bajo en $\mathrm{T} 1$, contrariamente al valor de $\mathrm{P}_{2} \mathrm{O}_{5}(0.92 \%)$ el cual es mayor en este tratamiento. Es importante notar la relación de $\mathrm{C} / \mathrm{N}$ en $\mathrm{T} 1$, la cual es casi tres veces más alta que el valor que más se le aproxima (T3).

Tabla 3 Número de bacterias y hongos (Penicillium y Aspergillus) por gramo de compost según cada tratamiento

\begin{tabular}{cccc}
\hline Tratamiento & $\begin{array}{c}\text { Bacterias (UFC/g de } \\
\text { compost) }\end{array}$ & $\begin{array}{c}\text { Penicillium (UFC/g de } \\
\text { compost) }\end{array}$ & $\begin{array}{c}\text { Aspergillus (UFC/g de } \\
\text { compost) }\end{array}$ \\
\hline T1 & $1.8 \times 10^{6}$ & 1 & 15 \\
T2 & $8 \times 10^{3}$ & 25 & 2 \\
T3 & $1.5 \times 10^{3}$ & 45 & 32 \\
T4 & $1.8 \times 10^{3}$ & 12 & 6 \\
T5 & $8 \times 10^{3}$ & 35 & 29 \\
\hline
\end{tabular}

Tabla 4 Coeficiente de correlación de Pearson entre los microorganismos presentes y la variable número de plantas emergidas

\begin{tabular}{lcc}
\hline & \multicolumn{2}{c}{ Coeficiente de correlación de PEARSON } \\
\hline \multirow{2}{*}{ Bacterias } & A. altilis & T. cacao \\
Penicillium & 0.79 & 0.48 \\
Aspergillus & 0.67 & 0.61 \\
& 0.71 & 0.64 \\
\hline
\end{tabular}

Los tratamientos T2 y T5 mostraron la mayor cantidad de bacterias y la mayor cantidad de hongos se reporta en el T3 (Tabla 3). En la tabla 4 se pueden observar los datos del coeficiente de correlación de Pearson ente los microorganismos presentes y la variable número de plantas emergidas en los tratamientos utilizados. En frutepan (A. altilis) se puede notar que las bacterias presentan el más alto coeficiente de correlación, mientras que en cacao, apa- rentemente, son los hongos, los que muestran el más alto coeficiente.

Las figuras 1, 2 y 3 , indican el comportamiento de las semillas de A. altilis y T. cacao, según cada tratamiento empleado en los compost donde germinaron. De manera general, se puede observar, que para cada parámetro medido (Días a la germinación, Fig.1; Plantas emergidas, Fig.2 y días al trasplante, Fig. 3), los mejores tratamientos son T3 y T4. Las 
semillas de frutepan se muestran bastante más tardías para germinar que las de cacao.

Figura 1 Días a la germinación de semillas de A. altilis y T. cacao, según cada tratamiento aplicado

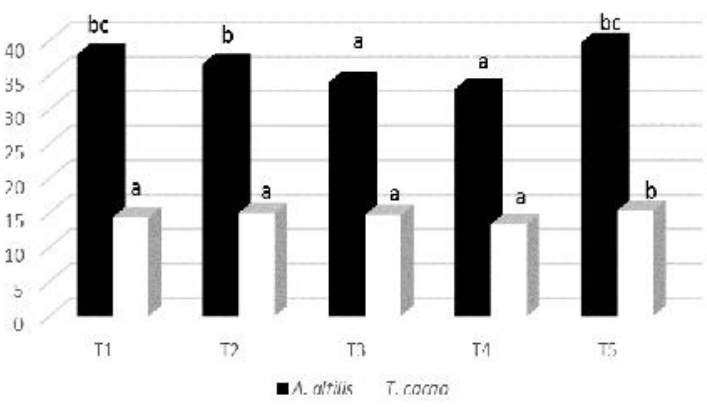

Figura 2 Total de plantas emergidas de semillas de $A$. altilis y $T$. cacao, según cada tratamiento aplicado

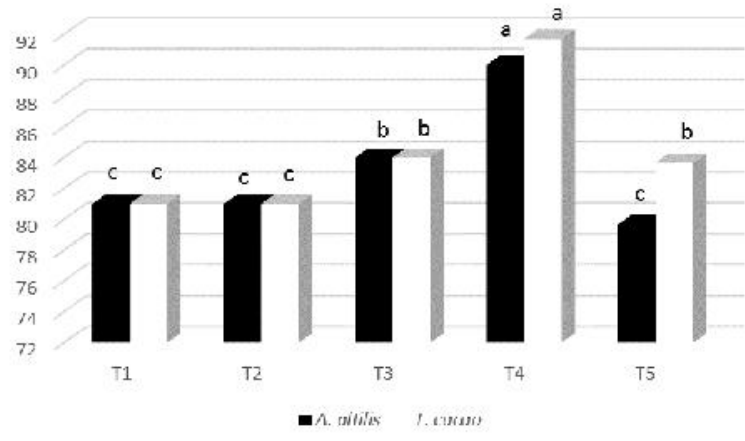

Figura 3 Días al trasplante de plantas emergidas de semillas de A. altilis y $T$. cacao, según cada tratamiento aplicado

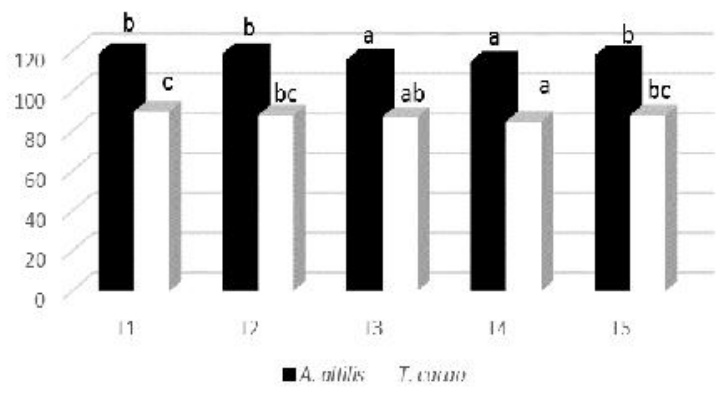

\section{Discusión}

En general los procesos de descomposición de residuos están mediados por la actividad de los microorganismos (Boulter et al. 2000). Las diferencias observadas entre los tratamientos, podrían deberse a la calidad de los materiales empleados, a las diferentes temperaturas durante el proceso de descomposición y a la cantidad de agua empleada. En el proceso de descomposición de la MO se presentan rangos de temperatura en los que predominan microorganismos diferentes. Temperaturas entre $45 \mathrm{y}$ $50{ }^{\circ} \mathrm{C}$ favorece el predominio de grupos de los aerobios mesofilicos y actinomycetes (Meléndez \& Soto 2003). Por el contrario, temperaturas superiores a $50{ }^{\circ} \mathrm{C}$ reducen las poblaciones de hongos (Tiquia et al. 2002).

En estas especies, parecieran ser muy importantes las diferencias observadas entre los tratamientos, sobre todo porque las semillas parecieran ser más recalcitrantes a la hora de germinar. Las diferencias significativas observadas en las semillas del cultivo de cacao, indican que cualquiera de los tratamientos utilizados, es más eficiente que el testigo con diferencias de hasta dos días para la germinación en relación a este último. El hecho de que las semillas de las especies seleccionadas no toleren la desecación, da un gran valor a estudios de sustratos que sean factibles para su establecimiento. Se observa una diferencia importante en artocarpus (de hasta 6 días) entre el tratamiento $\mathrm{T} 4 \mathrm{y}$ el testigo (T5), no observándose diferencias significativas entre T3 y T4. El mayor número de plantas emergidas de ambos cultivos, se observó con T4. Igual respuesta se aprecia con la variable días al trasplante (Fig.3). Los resultados de las tres variables evaluadas, muestran mucha similitud en los comportamientos entre el tratamiento con levadura comercial (T3) y la mezcla 
de todos los compost enriquecidos (T4). En la figura 2 , se puede observar que el total de plantas emergidas es similar al del cacao, tanto con $\mathrm{T} 3$ como con $\mathrm{T} 4$, a pesar de que artocarpus tarda más en germinar (Fig.1). Estas diferencias pueden ser atribuidas a que los microorganismos proporcionan una rápida descomposición de macromoléculas, haciendo que los macro y micro nutrientes solubles estén disponibles por la rápida descomposición, la cual es causa directa de la hidrolización que realizan los microorganismos como funcionamiento normal de su metabolismo para la obtención de nutrientes (Higa 2013). Los valores de $\mathrm{pH}$ observados (6.50-6.70) facilitan la absorción del fosforo y la conservación de la humedad, favoreciendo a la planta para un mejor desarrollo (Campo-Martínez et al. 2014).

El tratamiento $\mathrm{T} 1$ de compost enriquecido con microorganismos del suelo capturados in situ, no muestra ningún beneficio a la germinación y días al trasplante de plantas de los dos cultivos seleccionados en relación con los otros compost enriquecidos, comportándose de manera desventajosa frente al testigo para el número de plantas emergidas de cacao. El tratamiento T2 (LAB) no es significativamente diferente de los demás tratamientos con respecto a la germinación del cacao, se ha reportado que los cultivos de LAB pueden ser usados en conjunción con otros nutrientes para tratar a las semillas antes de plantarlas (Ikeda et al. 2013). Este procedimiento, mejoraría la germinación y disminuiría las perdidas por patógenos en las semillas (Hamed et al. 2011).

En procedimientos de agricultura orgánica, los LAB se han usado con microorganismos indígenas (IMO) para hacer compost o preparar suelos antes de la siembra (Park \& DuPonte 2008). La aplicación de estas bacterias, adicionalmente, puede acelerar la descomposición de la materia orgánica e incremen- tar la liberación de otros nutrientes para su absorción por parte de las plantas (Higa \& Kinjo 1989).

\section{Conflictos de intereses}

Esta investigación se la realizó en el Centro de Investigación, Posgrado y Conservación Amazónica de la Universidad Estatal amazónica en la provincia de Pastaza Ecuador y no presenta conflictos de interés.

\section{Agradecimientos}

Los autores agradecen a la Universidad Técnica de Ambato, Facultad de Ciencias Agropecuarias y a la Universidad Estatal Amazónica, Centro de Investigación, Posgrado y conservación Amazónica CIPCA, por el apoyo técnico, científico y logístico realizado a la presente investigación.

\section{Literatura citada}

Benzing A. Agricultura Orgánica-fundamentos para la región andina. Neckar-Verlag, VillingenSchwenningen, Alemania. 2001; p. 682.

Boulter J, Trevors J, Boland G. Compost: A study of the development process and end-product potential for suppression of turfgrass disease. World J Microb Biot. 2000; 16:115-34.

Broomes J, Badrie N, Roberts-Nkrumah LB. Effect of cultivar and maturity on yield and nutrient composition of breadfruit (Artocarpus altilis) flour. Proceedings CFCS. 2009; 45: 128-35.

Caballero-Mellado J, Carcaño-Montiel MG, Mascarúa-Esparza MA. Field inoculation of wheat (Triticum aestivum) with Azospirillum brasilense under temperate climate. Symbiosis 1992; 13: 243-53. 
Campo-Martínez ADP, Acosta RL, Morales S, Prado FA. Evaluación de microorganismos de montaña (MM) en la producción de acelga en la meseta de Popayán. Rev Bio Agro. 2014; 12: 79-87.

Cañadas-Cruz, L. El mapa bioclimático y ecológico del Ecuador. Ministerio de Agricultura y Ganadería. Programa Nacional de Regionalización Agraria. Banco Central del Ecuador. Quito. 1988 .

Cassán FD, Garcia de Salamone I (eds). Azospirillum sp.: cell physiology, plant interactions and agronomic research in Argentina. I International Workshop on Azospirillum; Oct 12-13; Córdoba, Argentina: Asociación Argentina de Microbiologia; 2008.

Changrun L, Wendell QS. Desiccation sensitivity and activities of free radical-scavenging enzymes in recalcitrant Theobroma cacao seeds. Seed Sci Res 2009; 9: 209-17.

De Felipe-Antón MR. Interacciones microorganismos-suelo-planta en la preservación del Medio Ambiente y la Salud. An R Acad Nac Farm. 2004; 70: 743-76.

Díaz-Zorita M, Fernandez-Canigia MV. Field performance of a liquid formulation of Azospirillum brasilense on dryland wheat productivity. Eur J Soil Biol. 2009; 45:3-11.

Elein A, Leyva A, Hernández A. Microorganismos benéficos como biofertilizantes efecientes para el cultivo del tomate (Lycopersicon esculentum, Mill) An R Acad Nac Farm. 2005; 2: 47-54.

Fennah RG, Murray DB. The cocoa tree in relation to its environment. Cocoa conference. Londres. 1957. p. 222-7.
GAD, Santa Clara. Diagnóstico Ecoturístico del Cantón Santa Clara. Ecuador. Provincia de Pastaza. 2009.

García de Salamone I. Técnicas aplicadas al estudio de la diversidad microbiana del suelo. XII Congreso Ecuatoriano de la Ciencia del Suelo 2010 Nov 17-19; Santo Domingo: Sociedad Ecuatoriana de Ciencias del Suelo; 2010.

Grandy AS, Robertson GP, Thelen KD. Do productivity and environmental trade-offs justify periodically cultivating No-till cropping systems?. Agron J. 2006; 98:1377-83.

Hamed, HA, Moustafa YA, Abdel-Aziz SM. In vivo efficacy of lactic acid bacteria in biological control against Fusarium oxysporum for protection of tomato plant. Life Sci J. 2011; $8: 462-8$.

Higa T, Kinjo S. Effect of lactic acid fermentation bacteria on plant growth and soil humus formation. First International Conference on Kyusei Nature Farming, Khon Kaen, Thailand. 1989. Retrieved from http://infrc.or.jp/english /KNF_Data_Base_Web/1st_Conf_S_5_4.html.

Higa T. Microorganismos Benéficos y efectivos para una agricultura y medio ambiente sostenibles. Maryland (USA): Centro internacional de Investigación de Agricultura Natural, Departamento de Agricultura de los Estados Unidos, 2013. p. 13.

Ikeda DM, Weinert E, Chang KCS, McGinn JM, Miller SA, Keliihoomalu C, et al. Natural Farming: Lactic acid bacteria. Sustain Agric. 2013; 8:3-4.

Jagpat UB, Bapat VA. Artocarpus: A review of its traditional uses, phytochemistry and pharmacology. J Ethnopharmacol. 2010; 129(2): 14266. 
Jones AMP, Ragone D, Tavana NG, Bernotas DW, Murch SJ, Murch SJ. Beyond the Bounty: Breadfruit (Artocarpus altilis) for food security and novel foods in the 21st Century. Ethn Res Appl 2011; 9: 129-49.

Meléndez G, Soto G. Taller de Abonos Orgánicos: El Proceso de Compostaje. El proyecto NOS del CATIE/GTZ, el Centro de Investigaciones Agronómicas de la Universidad de Costa Rica y la Cámara de Insumos Agropecuarios No Sintéticos. . 2003: 30-55.

Navia-Cuetia CA, Zemanate-Cordoba Y, MoralesVelasco S, Prado FA, Albán-López N. Evaluación de diferentes formulaciones de compostaje a partir de residuos de cosecha de tomate (Solanum lycopersicum). Rev Bio Agro. 2013; npe(2): 165-73.

Park H, DuPonte MW. How to cultivate indigenous microorganisms. BIO-9; 1-6.

Pedraza RO, Teixeira KSA, Fernández-Scanvino A, Garcia I, Vaca BE, Azcón R, et al. Microorganismos que mejoran el crecimiento de las plantas y la calidad de los suelos. Revisión. Corpoica Cienc Tecnol Agropecu. 2010; 11(2): 15564.
Pérez A, Céspedes C, Núñez P. Caracterización física-química y biológicas de enmiendas orgánicas aplicadas en la producción de cultivos en República Dominicana. Plant Nutr. 2008; 8 (4): 10-29.

Ragone D. Artocarpus altilis (breadnut). Moraceae (Mulberry family) Species Profiles for Pacific Island Agroforestry. 2006. p: 1-17.

Roberts-Nkrumah LB, Legall G. Breadfruit (Arcarpus altilis, Moraceae) and chataigne (A. camansi) for food security and income generation: The case of Trinidad and Tobago. Econ Bot. 2013; 67: 324-34.

Tiquia S, Wan J, Tam n. Microbial population dynamics and enzyme activities during composting. Compost Sci Util. 2002; 10(2): 150-61.

Wang Y, Deng T, Lin L, Pan Y, Zheng X. Bioassay-guided Isolation of Antiatherosclerotic Phytochemicals from Artocarpus altilis. Phytother Res. 2006; 20(12): 1052-5. 